\title{
The Effect of Epidural Resiniferatoxin in the Neuropathic Pain Rat Model Randomized Trial: A Complementary Mechanism
}

\section{TO THE EDITOR:}

I read with great interest the paper by Lee et al titled "The Effect of Epidural Resiniferatoxin in the Neuropathic Pain Rat Model" (1). This work addressed that resiniferatoxin, which is a decoy receptor of transient receptor potential Vanilloid subtype 1, improved neuropathic pain. I would like to complete the discussion of Lee and colleagues by introducing a major complementary route which resiniferatoxin could reduce neuropathic pain.

The essential role of neuropeptides in mediating neuropathic pain is evidenced by many works $(2,3)$. Recent studies have shown that resiniferatoxin not only produces various antinociception factors, including
GMAP (galanin message-associated protein) and nitric oxide, but also is capable of down-regulation of neuropeptides such as substance $P(4,5)$. Therefore, these important mechanisms should be borne in mind as the major complementary mechanisms for resiniferatoxin reduced neuropathic pain.

\author{
Hamid Namazi, MD \\ Associate Professor \\ Shiraz University of Medical Sciences \\ Shiraz, Iran \\ E-mail: namazih@sums.ac.ir
}

\section{References}

1. Lee M G, Huh BK, Choi S S, Lee D K, Lim $B$ G, Lee M. The effect of epidural resiniferatoxin in the neuropathic pain rat model. Pain Physician 2012; 15:287-296.

2. Birklein F, Schmelz M. Neuropeptides, neurogenic inflammation and complex regional pain syndrome (CRPS). Neurosci Lett 2008; 437:199-202.

3. Fredberg $U$, Stengaard-Pedersen $K$.
Chronic tendinopathy tissue pathology, pain mechanisms, and etiology with a special focus on inflammation. Scand $] 5^{-}$. Med Sci Sports 2008; 18:3-15.

4. Farkas-Szallasi T, Lundberg JM, Wiesenfeld-Hallin Z, Hökfelt T, Szallasi A. Increased levels of GMAP, VIP and nitric oxide synthase, and their mRNAs, in lumbar dorsal root ganglia of the rat following systemic resiniferatoxin treatment. Neuroreport 1995; 6:2230-1124.

Yang X, Gong H, Liu Z, Liu H, Wang H, Li Z. Similar and different effects of capsaicin and resiniferatoxin on substance $P$ release and transient receptor potential vanilloid type 1 expression of cultured rat dorsal root ganglion neurons. Methods Find Exp Clin Pharmacol 2010; 32:3-11. 